\title{
The Preclinical Evaluation of Self-Replicating RNA Oral Vaccine to Simultaneously Neutralize Both SARS-CoV-2 Delta and Alpha Variants
}

\author{
Abbas Mohammadi \\ Sirjan School of Medical Sciences \\ Ali Jebali ( $\square$ alijebal2011@gmail.com ) \\ Islamic Azad University
}

\section{Research Article}

Keywords: Preclinical study, Self-replicating RNA, Vaccine, B.1.1.7 lineage, B.1.617 lineage, SARS-COV-2

Posted Date: August 5th, 2021

DOl: https://doi.org/10.21203/rs.3.rs-753957/v1

License: (c) (1) This work is licensed under a Creative Commons Attribution 4.0 International License.

Read Full License 


\section{Abstract}

The aim of this study was to design an oral vaccine based on self-replicating RNA lipid nanoparticle (saRNA LNP) to neutralize SARS-CoV-2 Delta (B.1.617 lineage) and Alpha (B.1.1.7 lineage) variants. Before immunization of mice with saRNA LNP, we saw the high expression of S-protein at both mRNA and protein levels after transfection of HEK293T/17 with saRNA LNP. After immunization of BALB/c mice with saRNA LNP at 0.1-10 $\mu$ g, a high quantity of SARS-CoV-2 specific $\lg G, \lg G 1, \lg G 2 a$, and $\lg A$ was seen with a dose-dependent and Th1/Th2 skewing response in serum of vaccinated mice. We also found that the secreted antibodies could neutralize SARS-CoV-2 Delta (B.1.617 lineage) and Alpha (B.1.1.7 lineage) variants. Splenocytes from vaccinated mice re-stimulated with SARS-CoV-2 peptides had high IFN-ץ, IL-6, and TNF- a secretion. Although the preclinical study confirmed the efficacy of saRNA LNP against SARSCoV-2, its efficacy and safety must be evaluated in clinical trials.

\section{Introduction}

Vaccines have different formulations and they are becoming more advanced every day ${ }^{1}$. The use of nucleic acids and nanomaterials are two advanced technologies, recently used in vaccines ${ }^{2}$. Early studies focused on the use of DNA instead of RNA because RNA is less stable than DNA. Since DNA vaccines have shown poor results in human clinical trials ${ }^{3}$, this led to the interest in using RNA. Of course, this change in strategy is partly attributed to the success of cancer immunotherapy by mRNA molecules ${ }^{4,5}$. There are currently two types of RNA vaccines, including conventional mRNA and selfreplicating RNA (saRNA) ${ }^{6}$. These RNAs are produced in vitro and encode pathogen antigens and this is proportional to the number of mRNA copies ${ }^{7}$.

To design a saRNA, the sequence of an RNA-dependent RNA polymerase (RdRP) complex from viral origin, as well as the $5^{\prime}$ and $3^{\prime}$ untranslated regions needed for replication are included in an RNA construct that also contains the gene of interest. In this way, RdRP will be able to amplify the saRNA in the cytoplasm ${ }^{8}$ and it can activate the immune system ${ }^{9}$. The production of saRNA vaccines is simple and cost-effective and can be easily integrated into a production line ${ }^{10}$. Hekele et al. showed that a saRNA vaccine for influenza H7N9 can be produced within eight days ${ }^{11}$. There are several formulations that can be used to deliver saRNA vaccines, including cationic polymer-based pABOLs ${ }^{12}$, mannosylated LNPs (MLNPs) ${ }^{13}$, and neutral LPPs ${ }^{14}$. Because of the self-replicating property of saRNA, a high immune response can be achieved with low doses ${ }^{15}$. The addition of mannose to the LNP formulation also improves the immune response ${ }^{13}$. Importantly, saRNA activity occurs in the cytoplasm and therefore does not require entry into the cell nucleus. Indeed, there is no risk of integration of saRNA into the genome due to its function in the cytoplasm $4,5,16$.

The aim of this study was to design an oral vaccine based on saRNA LNP to neutralize SARS-CoV-2 Delta (B.1.617 lineage) and Alpha (B.1.1.7 lineage) variants. 


\section{Materials And Methods \\ Plasmid construct}

To synthesize the saRNA construct, a mammalian plasmid vector, pcDNA3.1, was used with four main parts, including 1) the sub-genomic CMV promoter, 2) the NSP sequence of Trinidad donkey Venezuelan equine encephalitis virus (GenBank accession number: NC_001449) required for replication, 2) the sequence of Spike protein of SARS-COV-2 variant B.1.1.7 lineage (GenBank accession number: MW735440), and the sequence of Spike protein of SARS-COV-2 variant B.1.617 lineage (GenBank accession number: MZ149976). The construct was synthesized and cloned by Biomatik, Canada into pcDNA3.1 plasmid. Positive control plasmid was the same vector containing only the sequence of Spike protein of SARS-COV-2 (variants B.1.1.7 and B.1.617) without the sequence of Trinidad donkey Venezuelan equine encephalitis virus. And, negative control plasmid was pcDNA3.1 plasmid without the sequence of Spike protein of SARS-COV-2 and the sequence Trinidad donkey Venezuelan equine encephalitis virus. Figure 1 a shows the diagrams of saRNA plasmid, positive control plasmid, and negative control plasmid. Figure $1 \mathrm{~b}$ shows the diagrams of linear saRNA and its FASTA sequence is in supplementary 1 .

\section{Synthesis of linear saRNA}

The sequence of saRNA was first replicated by simple PCR in presence of PFU DNA polymerase (SigmaAldrich) and saRNA plasmid as a DNA template. The replicated DNA was purified by a Plasmid Plus MaxiPrep kit (QIAGEN, UK) and its purity was determined by a NanoDrop (ThermoFisher, UK). Next, the purified DNA was first reacted with MEGAScript ${ }^{\mathrm{TM}}$ (Ambion, UK) and then reacted with ScriptCap ${ }^{\mathrm{TM}}$ (CellScript, WI, USA) for $1 \mathrm{~h}$ at $37^{\circ} \mathrm{C}$. Then, synthesized saRNA was purified by LiCl precipitation, resuspended in RNA storage buffer, and stored at $-80^{\circ} \mathrm{C}$.

\section{Encapsulation of saRNA in lipid nanoparticles (LNP)}

To encapsulate saRNA, we used a simple chemical process in which $100 \mathrm{nM}$ purified saRNA at $\mathrm{pH}=4.0$ were mixed with an ethanolic lipid mixture of 1,2-dilinoleyloxy-3-dimethylaminopropane, 1,2diastearoylsn-glycero-3-phosphocholine, cholesterol, and PEG-DMG 2000 at a ratio of 10:48:2:40. The mixture was stirred vigorously by T-mixer and then placed in a dialysis bag to purify overnight. The size distribution of the produced LNP was determined by a dynamic light scattering (DLS) (Malvern Instruments Ltd, Malvern, UK). Also, the encapsulation of saRNA was confirmed by gel electrophoresis and the entrapment efficiency was measured by a NanoDrop (ThermoFisher, UK) at $260 \mathrm{~nm}$. For positive and negative control plasmids, the same encapsulation process was also carried out.

\section{The expression of saRNA in HEK293T/17 cells}

HEK293T/17 cells were first cultured in complete Dulbecco's Modified Eagle's Medium (DMEM) (Gibco) containing 10\% fetal bovine serum (FBS) (Gibco), 1\% L-glutamine (Thermo Fisher Scientific), and 1\% penicillin-streptomycin (Thermo Fisher Scientific) at $37^{\circ} \mathrm{C}$ and $5 \% \mathrm{CO}^{2}$. The cultured cells were separately 
transfected by $0.1,1$, and $10 \mu \mathrm{g}$ saRNA LNP, positive control LNP, and negative control LNP. After 48 hours, the expression of S-protein at mRNA level was investigated by Q-PCR, and the expression of Sprotein at protein level was determined by an ELISA kit.

\section{Immunization}

All procedures related to animal experiments used in this section of the study were approved by an animal ethical committee of Sirjan University of Medical Sciences, Sirjan, Iran. Here, we used 10 BALB/c mice aged 6-8 weeks for each group study. In the main study groups, BALB/c mice were orally immunized with $0.1,1$, and $10 \mu \mathrm{g}$ saRNA LNP at weeks 1 and 3 . The volume of vaccine used for each mouse was $100 \mu \mathrm{L}$ and vaccine administration was done using a needleless insulin syringe. In the control groups, BALB/c mice were orally immunized with $10 \mu \mathrm{g}$ positive control LNPs and $10 \mu \mathrm{g}$ negative control LNPs at weeks 1 and 3. Serum samples were collected from all mice at two-week intervals and the spleens were removed at week 6.

\section{Recovered COVID-19 patient samples}

Here, the serum samples of recovered COVID-19 patients $(n=10)$ were collected from Emam hospital, Tehran University of Medical Sciences, Tehran, Iran following written informed consent. All recovered patients had PCR-negative at the time of sampling.

\section{The serum level of $\mathrm{lgG} / \lg \mathrm{G} 1 / \lg \mathrm{g} 2 \mathrm{a} / \mathrm{lgA}$ in mice and recovered COVID-19 patients}

Here, a semi-quantitative ELISA was used to detect the level of IgG/lgG1/lgG2a/lgA in the sera. First, high binding ELISA plates (Biomat, Italy) were coated with SARS-CoV-2 Spike Protein Recombinant Antigen (Sigma-Aldrich) at $1 \mu \mathrm{gmL}^{-1}$. After washing the plates, $50 \mu \mathrm{L}$ of diluted serum samples collected from immunized mice and recovered COVID-19 patients were separately added. After incubation for $1 \mathrm{~h}$ at $37^{\circ} \mathrm{C}$, plates were washed and then $100 \mu \mathrm{L}$ of secondary antibody was added. The secondary antibodies used in this study were: 1) anti-mouse IgG-HRP, 2) anti-mouse IgG1-HRP, 3) anti-mouse IgG2a-HRP, 4) antimouse IgA-HRP, 5) anti-human IgG-HRP, 6) anti-human IgG1-HRP, 7) anti-human IgG2a-HRP, 8) anti-human IgA-HRP (Southern Biotech). After incubation and washes, $50 \mu \mathrm{L}$ of 3,3', 5,5'-tetramethylbenzidine was added and then stopped by a stopping solution. Finally, the absorbance of each well was read by a Spectrophotometer at $450 \mathrm{~nm}$ (BioTek Industries).

\section{Viral neutralization assay}

Serum samples from immunized mice and recovered COVID-19 patients were separately incubated with both SARS-CoV-2 variant B.1.1.7 and variant B.1.617 for $1 \mathrm{~h}$ at room temperature. These two variants were obtained from COVID-19 patients who had been hospitalized and confirmed by PCR. Then, the mixture was transferred to 96-well plates pre-seeded with Vero-E6 cells cultured in DMEM (Gibco) containing 10\% FBS (Gibco), 1\% L-glutamine (Thermo Fisher Scientific), and 1\% penicillin-streptomycin (Thermo Fisher Scientific). After incubation at $37^{\circ} \mathrm{C}$ and $5 \% \mathrm{CO}^{2}$ for 5 days, crystal violet $5 \%$ was added 
and they were scored for cytopathic effect. Finally, a neutralization titer was calculated for each study group.

\section{IFN-Y ELISpots}

Based on the Mouse IFN-y ELISpotPLUS kit (Mabtech), anti-IFN-ץ pre-coated plates were first blocked with 10\% FBS, and then $2.5 \times 10^{5}$ mouse splenocytes and SARS-CoV-2 peptides (AGX819, Sigma-Aldrich) at 1 $\mu \mathrm{gmL}^{-1}$ were added. Plates were incubated overnight at $37^{\circ} \mathrm{C}$ and $5 \% \mathrm{CO}^{2}$. After incubation, biotinylated cytokine-specific detection antibodies and then streptavidin-enzyme conjugate and substrate were added. Finally, each well was investigated under a dissection microscope (Zeiss, Germany) and the number of secreting cells was calculated.

\section{The secretion of IL- 6 and TNF- $a$ in the supernatant of activated splenocytes and sera}

For this section, we used serum samples from immunized mice, serum samples from recovered COVID-19 patients, and the supernatant of activated mouse splenocytes with SARS-CoV-2 peptide pools (SigmaAldrich) at $1 \mathrm{\mu gmL}^{-1}$. To detect the level of IL-6 and TNF-a, a semi-quantitative ELISA was used. Briefly, a high binding ELISA plate (Biomat, Italy) was separately coated with anti-mouse IL-6 and TNF-a (Southern Biotech) and then samples were separately added. After incubation for $1 \mathrm{~h}$ at $37^{\circ} \mathrm{C}$, plates were washed, and then $100 \mu \mathrm{L}$ of secondary antibodies, including anti-mouse IL-6-HRP and TNF-a-HRP (Southern Biotech), were added. Then, $50 \mu \mathrm{L}$ of 3,3', 5,5'-tetramethylbenzidine was added, and then a stopping solution was added. Finally, the absorbance of each well was read by a Spectrophotometer at $450 \mathrm{~nm}$ (BioTek Industries).

\section{Statistical analysis}

GraphPad Prism (version 8.4) was used to prepare graphs and statistics. Here, one-way ANOVA was used to indicate significance at $P<0.05$ with $n=10$ biologically independent mice and $n=10$ recovered COVID19 patients. All graphs and data are shown as Mean \pm SD.

\section{Results}

\section{LNP characterization}

After synthesis of linear saRNA, they were encapsulated in LNP. The average particle size (Fig. 1c) and zeta potential (Fig. 1d) of saRNA LNP were $100 \pm 5 \mathrm{~nm}$ and $+17 \pm 0.6 \mathrm{mV}$, respectively. The highest entrapment efficiency of saRNA was near $67 \pm 2 \%$.

\section{The expression of S-protein}


Before immunization of mice with saRNA LNP, its efficacy was checked on HEK293T/17 cells. We could see a high expression of S-protein at both mRNA and protein levels after transfection of HEK293T/17 with saRNA LNP (Fig. 1e and Fig. 1f). We found that the relative expression of S-protein was increased at both mRNA and protein levels with increasing of LNP dose.

\section{The serum level of IgG, IgG1, IgG2a, and IgA}

A high quantity of SARS-CoV-2 specific IgG, $\lg G 1$, IgG2a, and IgA was seen in a dose-responsive manner in serum of mice and recovered COVID-19 patients (Fig. 2a-c). Here, significant differences were found between mice immunized with $10 \mu \mathrm{g}$ of saRNA LNP and mice immunized with 1 and $0.1 \mu \mathrm{g}$ of saRNA LNP $(P<0.05)$. Also, significant differences were seen between recovered COVID-19 patients and mice immunized with 1 and $0.1 \mu \mathrm{g}$ of saRNA LNP $(P<0.05)$. A Th1/Th2 skewing response (Fig. $2 \mathrm{~d})$ was also seen in mice vaccinated with positive control and saRNA LNP at 0.1-10 $\mu \mathrm{g}$. It means that all mice vaccinated with saRNA LNP and positive control LNP led to a Th1-biased response.

\section{Viral neutralization assay}

We observed a high viral neutralization titer in mice vaccinated with saRNA LNP at 0.1-10 $\mu$ g in a linear dose-dependent manner. We also found that the secreted antibodies induced by saRNA LNP could neutralize both SARS-CoV-2 variants B.1.1.7 and variant B.1.617 (Fig. 3a-b). Interestingly, secreted antibodies detected in recovered COVID-19 patients could neutralize both variants. A significant positive correlation was seen between SARS-CoV-2 specific IgG and SARS-CoV-2 neutralization titer in both mice vaccinated with saRNA LNP and recovered COVID-19 patients (Fig. 3c-d). This indicates that high antibody titers enable efficient viral neutralization.

\section{Cellular and cytokine responses}

We found that splenocytes from vaccinated mice re-stimulated with SARS-CoV-2 peptides had a high IFNy secretion in a linear dose-dependent manner (Fig. 4a). There was a significant difference between mice vaccinated with 1 or $10 \mu \mathrm{g}$ saRNA LNP and other vaccinated groups $(P<0.05)$. We also checked the secretion of IL- 6 and TNF-a by re-stimulated splenocyte and their serum level in vaccinated mice. It was shown that in both supernatants of re-stimulated splenocytes and vaccinated mouse sera, there was a high level of IL- 6 and TNF-a in a dose-dependent manner (Fig. 4b-e). Here, significant differences were seen between mice vaccinated with $10 \mu \mathrm{g}$ saRNA LNP and other vaccinated groups $(P<0.05)$. We did not detect a significant level of IL- 6 and TNF-a in the serum of recovered COVID-19 patients compared with vaccinated mice.

\section{Discussion}

In this study, we sought to develop a saRNA-based vaccine that could simultaneously fight both English (B.1.1.7) and Indian (B.1.617) varieties of SARS-COV-2. Vaccines based on saRNA have already been used for some infectious diseases ${ }^{11,17}$. This technology has also been used for COVID-19, showing some 
advantages versus conventional mRNA ${ }^{18,19}$. The strength of saRNA-based vaccines is that they can induce potent immune responses at low doses because of their self-replicating property. Of course, these vaccines, like other vaccines, can cause side effects and must be evaluated. Currently, various vaccines against SARS-COV-2 are designed with different platforms and almost all countries are using these approved vaccines ${ }^{20}$. The problem is that the genomic structure of SARS-COV- 2 is not stable and it is susceptible to genetic mutations ${ }^{21}$. This phenomenon leads to a significant reduction in the effectiveness of vaccines with any platform ${ }^{22}$. To overcome this problem, we hypothesized that a saRNA construct able to express 2 types of S-protein from important variants, like B.1.1.7 and B.1.617, could confer good protection against both of them. In this study, our saRNA includes the alphavirus replicase as well as the genes coding for of S-protein from B.1.1.7 and B.1.617 SARS-CoV-2 variants. The replicase is required for saRNA amplification and mediates to the production of many copies of this RNA, as well as of the subgenomic RNAs coding for the spike proteins in the cytoplasm ${ }^{23}$. This phenomenon leads to the production of a large number of S-proteins that could stimulate B cells and be utptaken by APCs leading to high humoral and cellular responses. Of course, it should be noted that saRNAs, in order to efficiently transferred to cells must be complexed to molecules that will both protect RNA and facilitate crossing the cell membrane and reaching the cytoplasm. One of the best carriers is LNPs ${ }^{18}$. We can easily change their surface charge or engineer their surface to target specific cells ${ }^{24}$. After synthesizing saRNA LNP, we first tested its efficacy on HEK293T cell line and then on mice. The high expression of S-protein at both mRNA and protein levels was seen after transfection of HEK293T/17 with saRNA LNP. This valuable finding gave us hope that the LNP saRNA might stimulate the immune system and could act as an effective vaccine. In this study, the vaccine was orally administered because our goal was to stimulate the immune cells located in the mucosa. Oral administration is much easier than injectable vaccines and is especially more acceptable in children. After vaccination of mice with saRNA LNP, we saw a high level of IgG, IgG1, IgG2a, and IgA against SARS-CoV-2 B.1.1.7 and B.1.617 variants in a dose-dependent manner. Interestingly, the concentration of $\operatorname{lgG} / \lg A$ in the serum of recovered COVID-19 patients was approximately equal to their concentration in the serum of mice vaccinated with saRNA LNP at $10 \mu \mathrm{g}$. Another important finding was Th1/Th2 skewing or Th1-biased response. Moreover, a high viral neutralization titer was seen in mice vaccinated with saRNA LNP at 0.1-10 $\mu \mathrm{g}$ and in recovered COVID-19 patients. Interestingly, these antibodies could neutralize both variants of SARS-CoV-2 and this shows that the designed system (saRNA LNP) has fully worked and has been able to overcome the mutations of these two variants. As a complementary test, the secretion of IFN-y, IL-6, and TNF-a was also examined and we found that splenocytes from vaccinated mice re-stimulated with SARS-CoV-2 peptides had a high secretion of IFN- $\gamma$, IL-6, and TNF- $a$. We could also detect a relatively high level of IL- 6 and TNF- $a$ in the serum of recovered COVID-19 patients.

Before us, McKay et al. had been shown that saRNA SARS-CoV-2 LNP vaccine induces remarkably high and dose-dependent SARS-CoV-2 specific antibody titers in mouse sera, as well as robust neutralization of both a pseudo-virus and wild-type virus ${ }^{18}$. Also, Spencer et al showed vaccination with saRNA and adenoviral COVID vaccines induce robust immune responses in mice. They demonstrated that two-dose heterologous vaccination was better than single-dose. Neutralizing titers after heterologous prime-boost 
were at least comparable or higher than the titers measured after homologous prime-boost vaccination with viral vectors ${ }^{19}$. It is important to know the intestinal immune responses against SARS-CoV-2 to develop more effective vaccines against the virus ${ }^{25}$. Some studies on COVID-19 animal models ${ }^{26}$ and COVID-19 patients with gastrointestinal symptoms ${ }^{27}$ revealed that intraepithelial CD $8+$ lymphocytes and lamina propria residing CD $4+$ and $C D 8+$ effector $T$ cells are significantly expanded compared with healthy controls ${ }^{28}$. Importantly, inflammatory dendritic cells are significantly reduced in the lamina propria of COVID-19 patients with gastrointestinal symptoms ${ }^{28}$. These data suggest that intestinal infection with SARS-CoV2 alters immune signatures and leads to a more favorable immune response. Interestingly, the serum levels of IL- 6 and IL-17 are lower in COVID-19 patients with GI symptoms compared with COVID-19 patients withut GI symptoms. Another interesting point is that the presence of the virus in GI tract may trigger a long-term production of anti-viral IgA antibodies, compared with IgG and $\lg A$ antibodies 29,30 .

Taken together, the oral vaccine, based on saRNA LNP, could stimulate a Th1-biased response to produce a high quantity of SARS-CoV-2 specific IgG, IgG1, IgG2a, and IgA. We also found that the produced antibodies induced by saRNA LNP could neutralize SARS-CoV-2 Delta (B.1.617 lineage) and Alpha (B.1.1.7 lineage) variants.

\section{Declarations}

\section{Study approval}

All participants provided written informed consent for participation in the study before enrollment. The study was approved by review board of Sirjan University of Medical Sciences, Sirjan, Iran.

\section{DATA AVAILABILITY}

The data that support the findings from this study are available from the corresponding author on reasonable request.

\section{Acknowledgments}

We also thank Dr. Cristian Smerdou (Cima Universidad de Navarra, Spain) and PDDr. Vladimir Temchura, Institute of Clinical and Molecular Virology, Friedrich-Alexander-University Erlangen-Nürnberg, 91054 Erlangen, Germany for valuable helps. We thank the Emam hospital, Tehran University of Medical Sciences, Tehran, Iran for collecting recovered COVID-19 patients. Also, we thank Zahedan University of Medical Sciences, Zahedan, Iran for the laboratory facility.

\section{Funding}

This article was financially supported by Sirjan University of Medical Sciences, Sirjan, Iran (grant number: 99000071). 


\section{Authors' contributions}

(I) Conception and design: A.M and A.J, (II) Administrative support: A.M and A.J, (III) Provision of study materials or patients: A.M, (IV) Collection and assembly of data: A.J, (V) Data analysis and interpretation: A.M and A.J, (VI) Manuscript writing: All authors, (VII) Final approval of manuscript: All authors.

\section{Ethical approval}

All experiments were under the guidelines of the National Institute of Health, the provisions of the Declaration of Helsinki, and the ethics committee of Sirjan University of Medical Sciences, Sirjan, Iran. (Ethical code: IR.SIRUMS.REC.1400.001).

\section{Competing interests}

The authors state no conflict of interest.

\section{Informed consent}

Informed consent was obtained from all individuals included in this study.

\section{Statement}

The study is in accordance with ARRIVE guidelines (https://arriveguidelines.org)

\section{References}

1. D’Amico, C., Fontana, F., Cheng, R. \& Santos, H. A. Development of vaccine formulations: past, present, and future. Drug Delivery and Translational Research11, 353-372 (2021).

2. Shen, Y., Hao, T., Ou, S., Hu, C. \& Chen, L. Applications and perspectives of nanomaterials in novel vaccine development. MedChemComm9, 226-238 (2018).

3. Hobernik, D. \& Bros, M. DNA vaccines-how far from clinical use? International journal of molecular sciences19, 3605 (2018).

4. Gilboa, E. \& Vieweg, J. Cancer immunotherapy with mRNA-transfected dendritic cells. Immunological reviews199, 251-263 (2004).

5. Schlake, T., Thess, A., Thran, M. \& Jordan, I. mRNA as novel technology for passive immunotherapy. Cellular and Molecular Life Sciences76, 301-328 (2019).

6. Verbeke, R., Lentacker, I., De Smedt, S. C. \& Dewitte, H. Three decades of messenger RNA vaccine development. Nano Today28, 100766 (2019). 
7. Fuller, D. H. \& Berglund, P. Amplifying RNA vaccine development. New England Journal of Medicine382, 2469-2471 (2020).

8. Ferrero, D., Ferrer-Orta, C. \& Verdaguer, N. Viral RNA-dependent RNA polymerases: a structural overview. Virus Protein and Nucleoprotein Complexes, 39-71 (2018).

9. Vogel, A. B. et al. Self-amplifying RNA vaccines give equivalent protection against influenza to mRNA vaccines but at much lower doses. Molecular Therapy26, 446-455 (2018).

10. Jackson, N. A., Kester, K. E., Casimiro, D., Gurunathan, S. \& DeRosa, F. The promise of mRNA vaccines: a biotech and industrial perspective. npj Vaccines5, 1-6 (2020).

11. Hekele, A. et al. Rapidly produced $S A M \circledast$ vaccine against $H 7 N 9$ influenza is immunogenic in mice. Emerging microbes \& infections2, 1-7 (2013).

12. Blakney, A. K. et al. Big is Beautiful: Enhanced saRNA delivery and immunogenicity by a higher molecular weight, bioreducible, cationic polymer. ACS nano14, 5711-5727 (2020).

13. Goswami, R. et al. Mannosylation of LNP results in improved potency for self-amplifying RNA (SAM) vaccines. ACS infectious diseases5, 1546-1558 (2019).

14. Perche, F. et al. Neutral lipopolyplexes for in vivo delivery of conventional and replicative RNA vaccine. Molecular Therapy-Nucleic Acids17, 767-775 (2019).

15. Lundstrom, K. The Potential of Self-amplifying RNA Vaccines for Infectious Diseases and COVID-19. Vaccine Research7, 25-37 (2020).

16. Blakney, A. K., Ip, S. \& Geall, A. J. An update on self-amplifying mRNA vaccine development. Vaccines9, 97 (2021).

17. Luisi, K. et al. Development of a potent Zika virus vaccine using self-amplifying messenger RNA. Science advances6, eaba5068 (2020).

18. McKay, P. F. et al. Self-amplifying RNA SARS-CoV-2 lipid nanoparticle vaccine candidate induces high neutralizing antibody titers in mice. Nature communications11, 1-7 (2020).

19. Spencer, A. J. et al. Heterologous vaccination regimens with self-amplifying RNA and adenoviral COVID vaccines induce robust immune responses in mice. Nature communications12, 1-8 (2021).

20. Li, Y. et al. A comprehensive review of the global efforts on COVID-19 vaccine development. ACS Central Science7, 512-533 (2021).

21. Na, W., Moon, H. \& Song, D. A comprehensive review of SARS-CoV-2 genetic mutations and lessons from animal coronavirus recombination in one health perspective. Journal of Microbiology59, 332-340 (2021). 
22. Noh, J. Y., Jeong, H. W. \& Shin, E.-C. SARS-CoV-2 mutations, vaccines, and immunity: implication of variants of concern. Signal Transduction and Targeted Therapy6, 1-2 (2021).

23. Kim, J., Eygeris, Y., Gupta, M. \& Sahay, G. Self-assembled mRNA vaccines. Advanced drug delivery reviews (2021).

24. Blakney, A. K., McKay, P. F., Yus, B. I., Aldon, Y. \& Shattock, R. J. Inside out: optimization of lipid nanoparticle formulations for exterior complexation and in vivo delivery of saRNA. Gene therapy 26, 363372 (2019).

25. Weidinger, C., Hegazy, A. N., Glauben, R. \& Siegmund, B. COVID-19-from mucosal immunology to IBD patients. Mucosal Immunology, 1-8 (2021).

26. Sia, S. F. et al. Pathogenesis and transmission of SARS-CoV-2 in golden hamsters. Nature583, 834838 (2020).

27. Leung, W. K. et al. Enteric involvement of severe acute respiratory syndrome-associated coronavirus infection. Gastroenterology125, 1011-1017 (2003).

28. Livanos, A. E. et al. Gastrointestinal involvement attenuates COVID-19 severity and mortality. MedRxiv (2020).

29. Gaebler, C. et al. Evolution of antibody immunity to SARS-CoV-2. Nature591, 639-644 (2021).

30. Wang, Z. et al. neutralization by dimeric IgA. Sci. Transl. Med13.

\section{Figures}



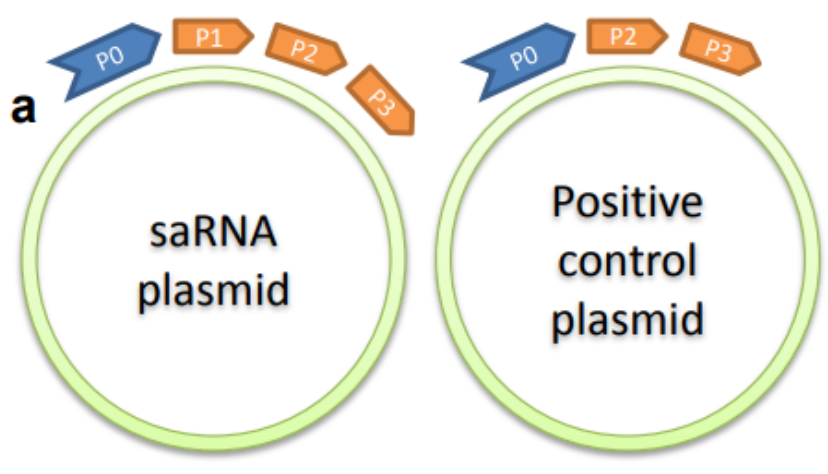

b
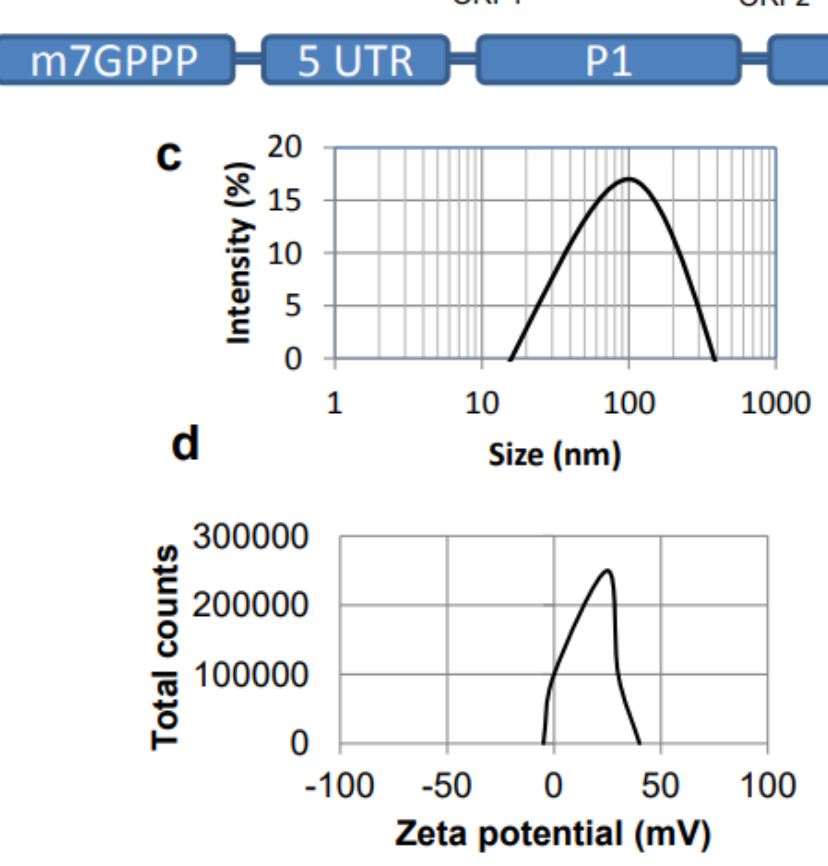
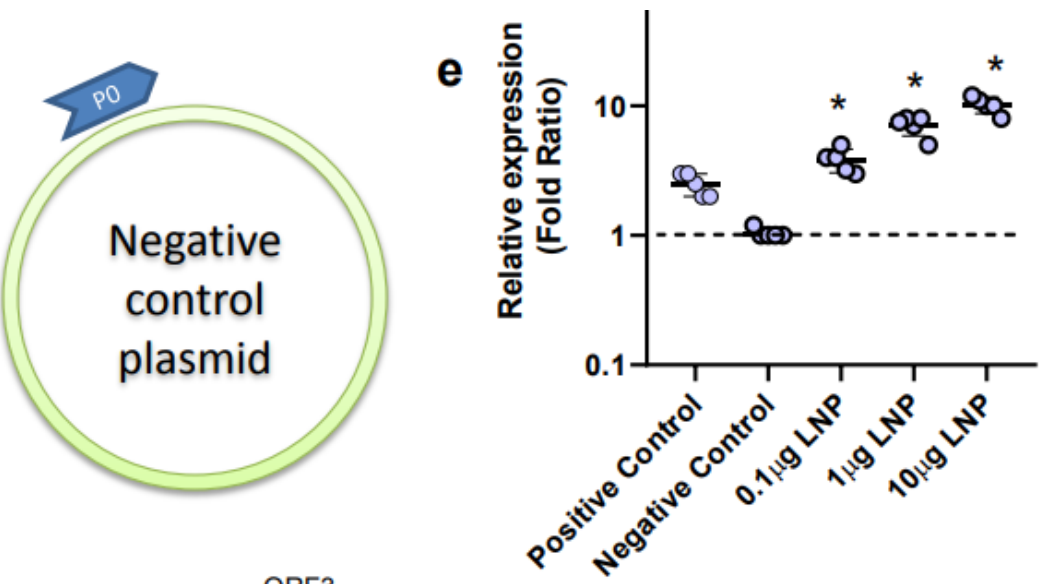

ORF3
P2

P3

3 UTR

Poly A

\section{Figure 1}

Diagrams of saRNA plasmid, positive control plasmid, and negative control plasmid (a) with 4 main parts, including P0) the sub-genomic CMV promoter, P1) the NSP sequence of Trinidad donkey Venezuelan equine encephalitis virus (GenBank accession number: NC_001449), P2) the sequence of Spike protein of SARS-COV-2 variant B.1.1.7 (GenBank accession number: MW735440), and P3) the sequence of Spike protein of SARS-COV-2 variant B.1.617 (GenBank accession number: MZ149976). Diagram of linear saRNA with different parts (b). The size distribution (c) and zeta potential (d) of saRNA LNP by DLS apparatus. The relative expression of S-protein at mRNA (e) and protein (f) level when HEK293T/17 cells treated with saRNA LNP at 0.1-10 $\mu \mathrm{g}$. indicates significance difference at $p<0.05$ when compared with negative control using a one-way ANOVA adjusted for multiple comparisons with $n$ $=5$. 
a

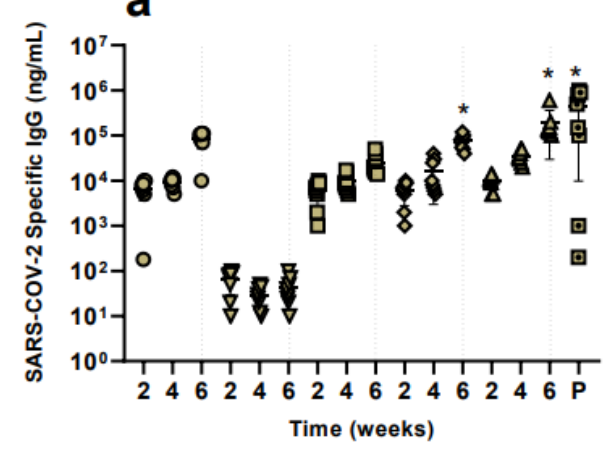

b

- Positive Control

$\nabla$ Negative Control

口 $0.1 \mu \mathrm{g}$ LNP

$\diamond 1 \mu \mathrm{g}$ LNP

$\Delta$ 10ug LNP

Recovered COVID-19

Patients

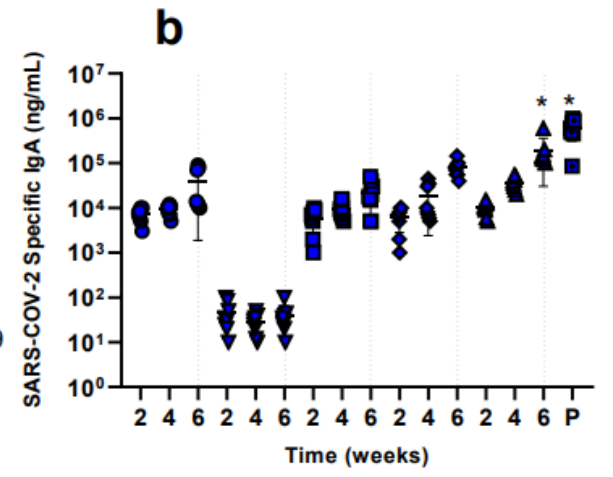

- Positive Control

v Negative Control

- $0.1 \mu \mathrm{g}$ LNP

- $1 \mu g$ LNP

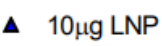

Recovered COVID-19 Patients
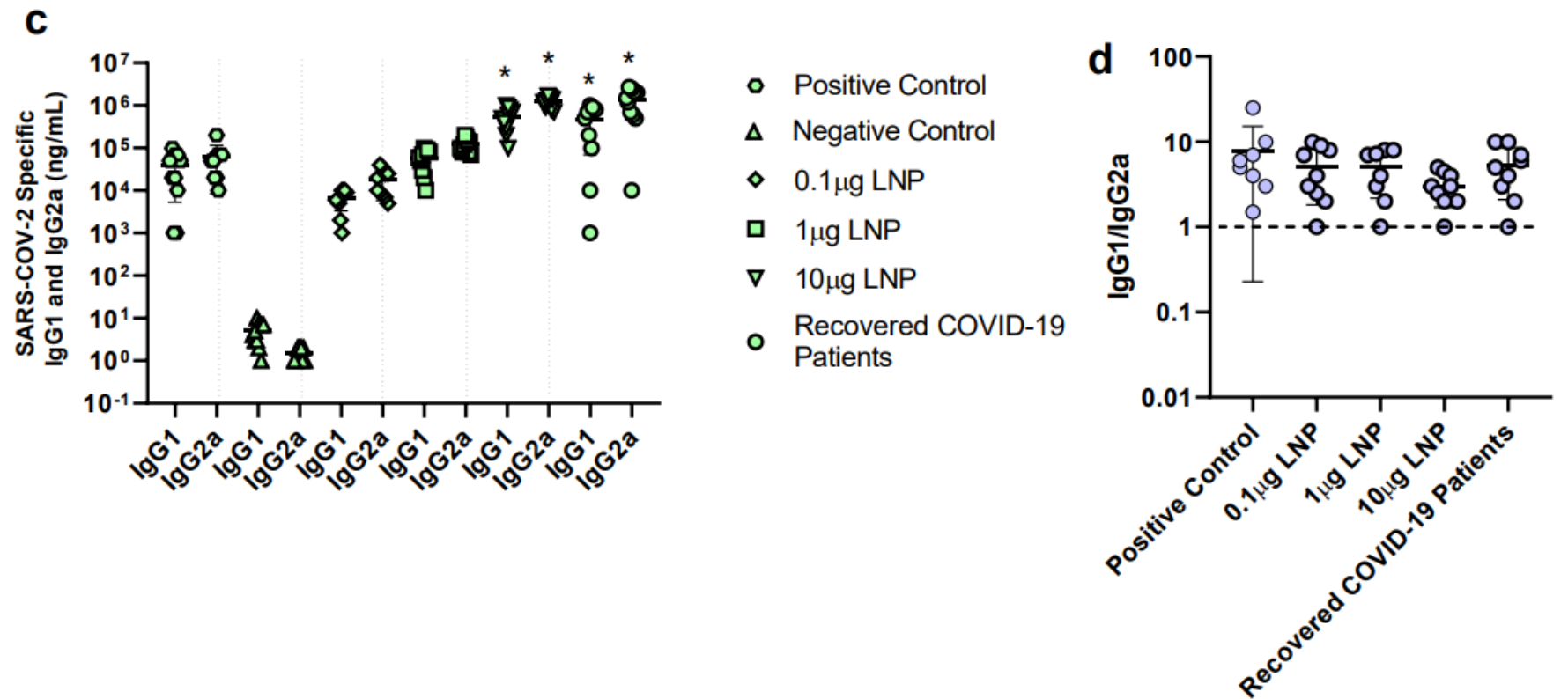

Figure 2

The serum level of IgG (a), IgA (b), IgG1 (c), and IgG2a (d) against SARS-CoV-2 in mice vaccinated with saRNA LNP at 0.1-10 $\mu \mathrm{g}$. Th1/Th2 skewing responses in vaccinated mice (e). * indicates significance difference at $p<0.05$ when compared with other groups using a one-way ANOVA adjusted for multiple comparisons with $n=10$ biologically independent mice and recovered COVID-19 patients. 


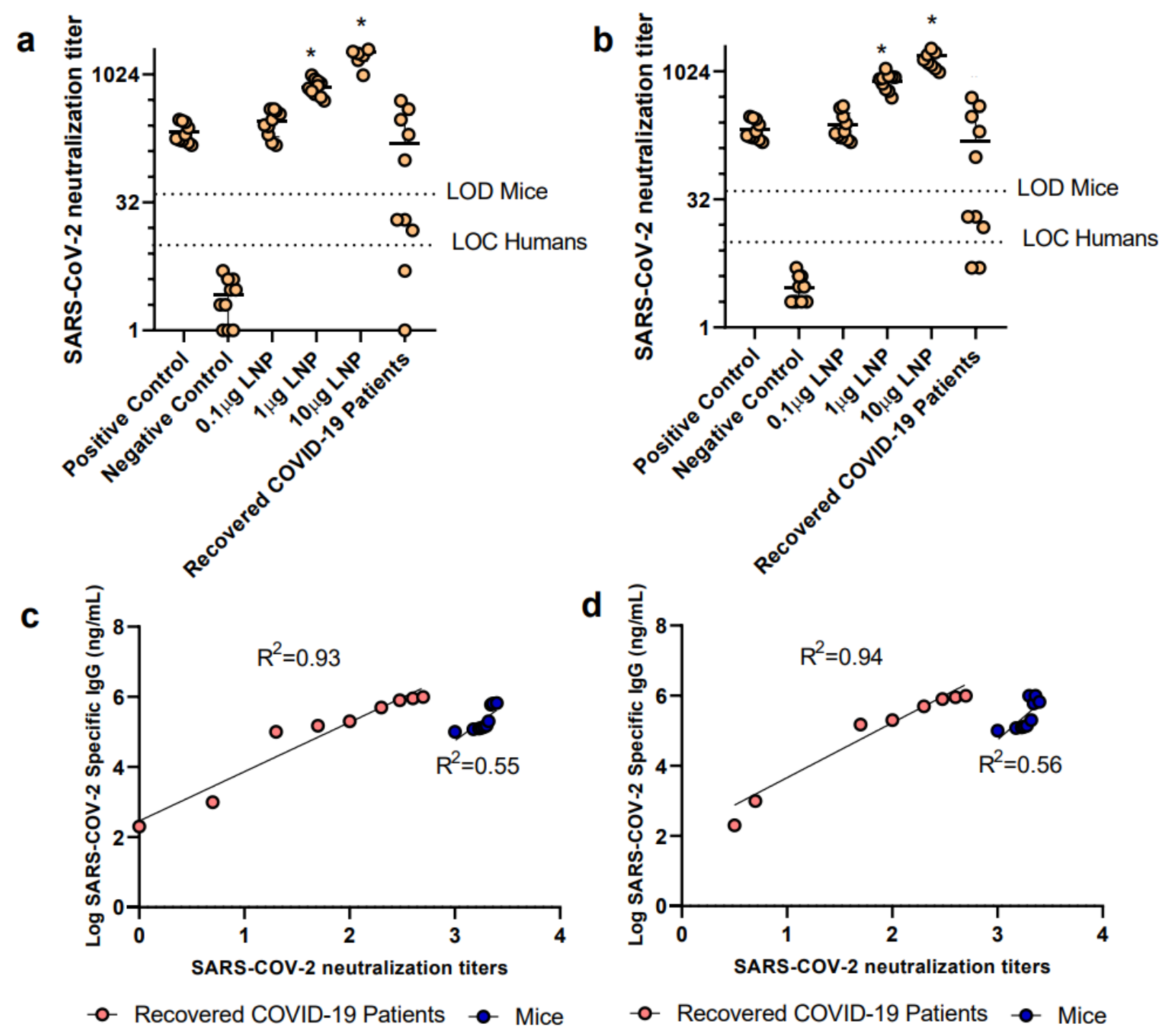

Figure 3

The viral neutralization titer of secreted antibodies against SARS-CoV-2 variant B.1.1.7 (a) and variant B.1.617 (b) in mice vaccinated with saRNA LNP at 0.1-10 $\mu \mathrm{g}$. A significant positive correlation was seen between SARS-CoV-2 specific IgG and SARS-CoV-2 neutralization titer of secreted antibodies against SARS-CoV-2 variant B.1.1.7 (c) and variant B.1.617 (d) in both vaccinated mice and recovered COVID-19 patients. * indicates significance difference at $p<0.05$ when compared with other groups using a oneway ANOVA adjusted for multiple comparisons with $n=10$ biologically independent mice and recovered COVID-19 patients. 


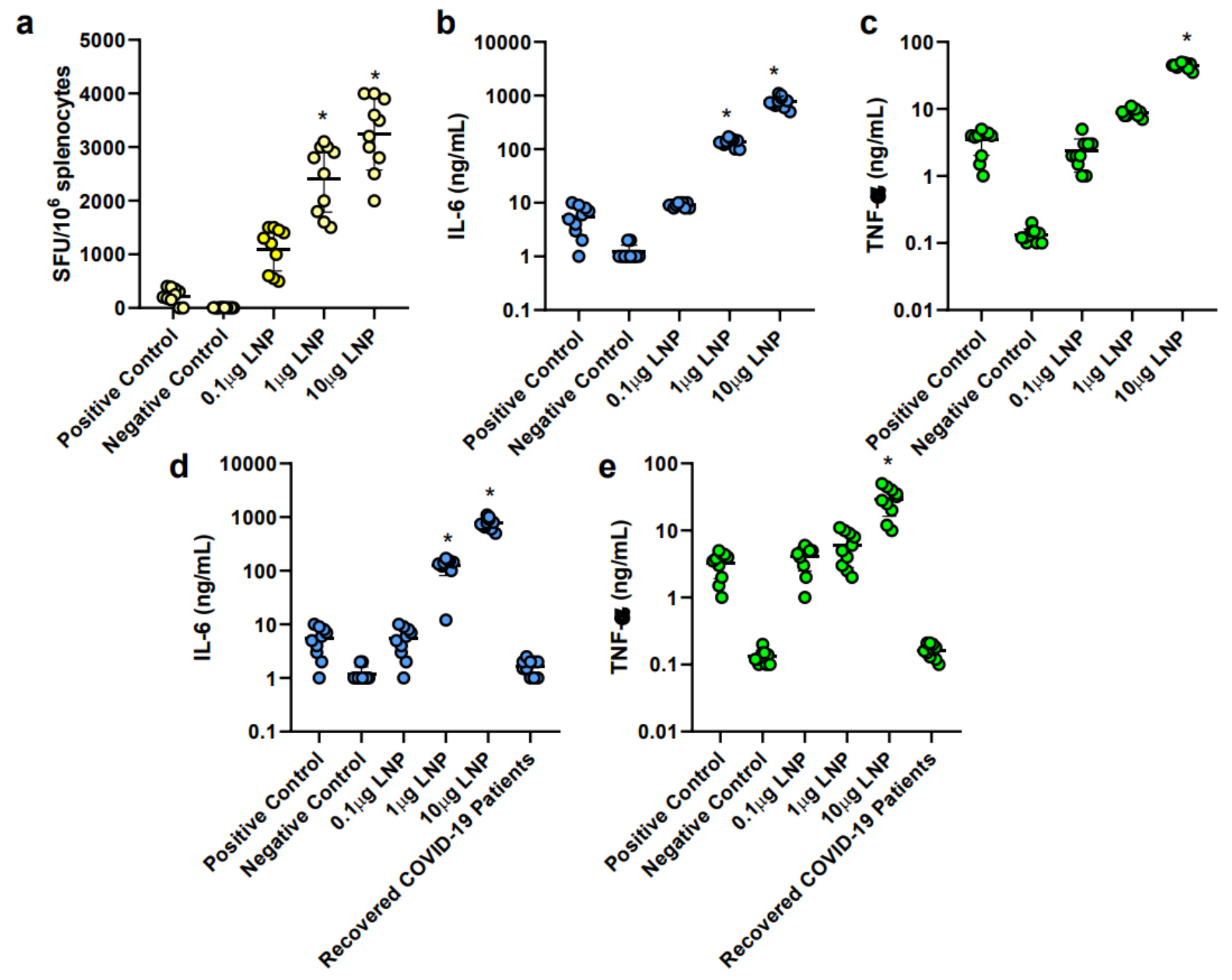

Figure 4

Splenocytes from vaccinated mice re-stimulated with SARS-CoV-2 peptides had a high IFN- $\gamma$ secretion in a linear dose-dependent manner (a). The secretion of IL-6 (b) and TNF-a (c) by re-stimulated splenocytes. The serum level of IL-6 (d) and TNF- $a$ (e) in mice vaccinated with saRNA LNP at 0.1-10 $\mu \mathrm{g}$ and in recovered COVID-19 patients. * indicates significance difference at $p<0.05$ when compared with other groups using a one-way ANOVA adjusted for multiple comparisons with $n=10$ biologically independent mice and recovered COVID-19 patients.

\section{Supplementary Files}

This is a list of supplementary files associated with this preprint. Click to download.

- supplementary1.docx 